\title{
Successful Pregnancy Outcome in Unicornuate Uterus: A Case Report
}

\author{
Feriha Fatima Khidri ${ }^{1}$, Faiza Kamran Ali ${ }^{2}$, Hafsa Shabir Ahmed ${ }^{3}$ \\ ${ }_{1}^{1}$ Lecturer, Department of Biochemistry, Liaquat University of Medical and Health Sciences, Jamshoro \\ 2 Postgraduate trainee Department of Gynaecology and Obstetrics, Liaquat University of Medical and Health sciences, Jamshoro \\ ${ }^{3}$ Postgraduate trainee Department of Gynaecology and Obstetrics, Dow University of Health Sciences - Ojha Campus
}

A B S TR ACT

Pregnancy with a unicornuate uterus is an infrequent obstetrical presentation. Unicornuate uterus leads to various complications, including infertility and poor pregnancy outcomes. It may be asymptomatic and remain undiagnosed till the woman is unable to conceive or present with a bad obstetric history. Here we present a case report of a patient with unicornuate uterus diagnosed during caesarean section along with delivery of alive baby.

Key Words: Outcome, Pregnancy, Unicornuate uterus

Address of Correspondence

Fariha Fatima

Email: ferihafatima@yahoo.com

Cite this case Report: Khidri FF, Ali FK, Ahmed HS. Successful Pregnancy Outcome in Unicornuate Uterus: A Casre Report. JIMDC. 2018; 7(4):307-308
Article info.

Received: November 9, 2018

Accepted: November 22, 2018

Funding Source: Nil

Conflict of Interest: Nil

\section{Introduction}

Congenital uterine anomalies are rare and unicornuate uterus is one of the infrequent presentations among uterine anomaly. The prevalence of a unicornuate uterus is found to be $0.3 \%$ among the general population, whereas it remains a cause of infertility in about $0.6 \%$ of the infertile women..$^{1,2}$ It usually remains undiagnosed till patient undergo certain surgical procedures or present with gynaecological, reproductive and obstetrical complications. It increases risks for abortion, infertility, preterm birth, premature rupture of membranes, abnormal lie, abnormal placental localization and intrauterine growth restrictions. $^{3}$

\section{Case Report}

A 30-year-old multigravida presented at the Liaquat University of Medical and Health sciences Hospital with 8 months' gestational amenorrhea and preterm premature rupture of membranes (PPROM). She had regular menstrual cycles and presented with history of recurrent fetal loss. The patient had one preterm baby delivered spontaneously vaginally, but the baby died in the early neonatal period, followed by two miscarriages at 2.5 months, two miscarriages at 4 months and one miscarriage at 4.5 months. After her last miscarriage, patient did not conceive for two years. On the advice of the local doctor, she took medications for ovulation induction and conceived after six months. At eight months of pregnancy she reported to a tertiary care hospital with complain of leaking. She received intramuscular dexamethasone cover at 33 weeks of gestation. Her baseline investigations were normal. She had normal vitals with reactive cardiotocography (CTG) on admission. After 48 hours, baseline rate dropped to $80 \mathrm{bpm}$, demonstrating pathological CTG for over 15 minutes. Caesarean section was performed due to fetal distress. While performing a caesarean section it was observed that the uterus was shifted from midline. Semilunar incision was given in the lower portion of the uterus and alive baby boy delivered in cephalic presentation. Placenta was expelled in $1 \mathrm{~min}$ and APGAR score was observed as 7 in one minute and 8 in 5 minutes. On examination unicornuate uterus was found with right fallopian tube normally located whereas left fallopian tube was located abnormally on the lower portion of the uterus 
(Figure 1). Left sided fallopian tube was thicker and originated anterioinferiorly on the lower portion of the uterus. Right side ovary was enlarged $7 \times 6 \mathrm{~cm}$ containing cyst whereas the left side ovary was normal in size but firm in consistency.
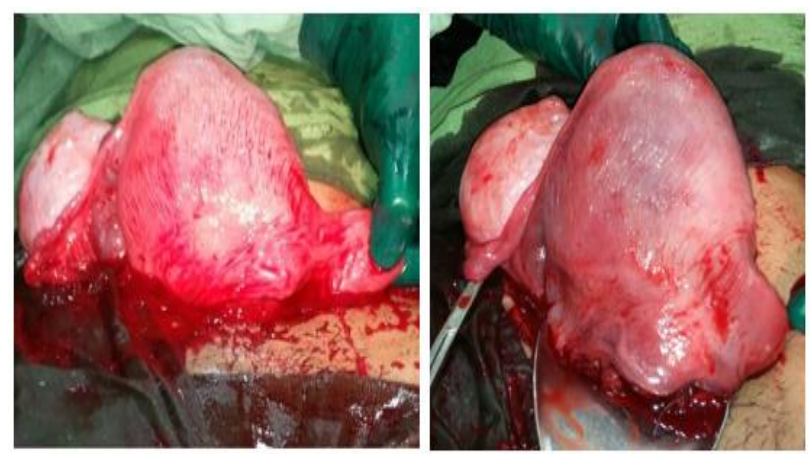

Figure 1a \& 1b: unicornuate uterus presentation in reported case

\section{Discussion}

Uterine anomalies are related to adverse pregnancy outcome and infertility. In the present case in spite of patient's bad obstetric history and hospitalization patient was unaware of structural anomaly and reasons for miscarriage. Although with the advancement in ultrasound technology, structural anomalies are easier to detect and diagnose, though it requires competent and experienced ultrasonologist and advanced ultrasound imaging and MRI technology. A normal ultrasound usually fails to rule out unicornuate uterus. Laparoscopy and hysteroscopy can detect unicornuate uterus; though, in this patient they were not advised, in spite of bad obstetric history. In the current case, uterine anomaly was diagnosed at the time of caesarean section, as only one ultrasound scan at the time of admission at 33 weeks' gestation was available; which did not detect anomaly. Heinonen PK in his retrospective study of uterine anomalies found right unicornuate uterus with non-communicating rudimentary horn as frequent type of presentation among uterine anomalies. ${ }^{4}$ Another research comprising 20 studies on the outcome of pregnancy in patients with unicornuate uterus revealed its incidence to be 1 in 4020 pregnancies. ${ }^{5}$ Unicornuate uterus predisposes women to greater risks for infertility, ectopic pregnancy, abortions, preterm delivery and intrauterine fetal death; where, only $50 \%$ of women are found to deliver alive babies. ${ }^{5}$ It is therefore suggested that women with bad obstetrical history and infertility should be considered for detailed anatomical evaluation of the uterus. Later these patients on conceiving should be considered high risk patients and managed accordingly.

\section{References}

1. Ombelet W, Verswijvel G, Vanholsbeke C, Schobbens JC. Unicornuate uterus and ectopic (undescended) ovary. Facts Views Vis Obgyn. 2011;3(2):131-134.

2. Rani A, Kumari M, Shipra. A case of noncommunicating uterine horn containing functional endometrium. Gynecology \& Obstetrics. 2015;5(9):320.

3. Caserta D, Mallozzi M, Meldolesi C, Bianchi P, Moscarini M. Pregnancy in a unicornuate uterus: a case report. J Med Case Rep. 2014;8(1):130.

4. Heinonen PK. Unicornuate uterus and rudimentary horn. Fertil Steril. 1997;68(2):224-230.

5. Reichman D, Laufer MR, Robinson BK. Pregnancy outcomes in unicornuate uteri: a review. Fertil Steril. 2009;91(5):1886-1894.. 